\title{
Receiver Orientation versus Transmitter Orientation in Linear MIMO Transmission Systems
}

\author{
Michael Meurer \\ Research Group for RF Communications, University of Kaiserslautern, P.O. Box 3049, 67653 Kaiserslautern, Germany \\ Email:meurer@rhrk.uni-kl.de \\ Paul Walter Baier \\ Research Group for RF Communications, University of Kaiserslautern, P.O. Box 3049, 67653 Kaiserslautern, Germany \\ Email:baier@rhrk.uni-kl.de \\ Wei Qiu \\ Research Group for RF Communications, University of Kaiserslautern, P.O. Box 3049, 67653 Kaiserslautern, Germany \\ Email:wqiu@rhrk.uni-kl.de
}

Received 23 June 2003; Revised 13 February 2004

\begin{abstract}
In conventional transmission schemes, the transmitter algorithms are a priori given, whereas the algorithms to be used by the receivers have to be a posteriori adapted. Such schemes can be termed transmitter (Tx) oriented and have the potential of simple transmitter implementations. The opposite to Tx orientation would be receiver $(\mathrm{Rx})$ orientation in which the receiver algorithms are a priori given, and the transmitter algorithms have to be a posteriori adapted. An advantage of the rationale Rx orientation is the possibility to arrive at simple receiver structures. In this paper, linear versions of the rationales Tx orientation and Rx orientation are applied to radio transmission systems with multiantennas both at the transmitter and receiver. After the introduction of adequate models for such multiple-input multiple-output (MIMO) systems, different system designs are evaluated by simulations, and recommendations for proper system solutions are given.
\end{abstract}

Keywords and phrases: MIMO systems, transmitter orientation, receiver orientation.

\section{INTRODUCTION}

In conventional transmission schemes the transmitter algorithms are a priori given and made known to the receiver, whereas the algorithms to be used by the receivers have to be a posteriori adapted, possibly under consideration of channel information. For this approach, where the transmitter $(\mathrm{Tx})$ is the master and the receiver $(\mathrm{Rx})$ is the slave, the authors propose the term Tx orientation. The opposite to Tx orientation would be Rx orientation in which the receiver algorithms would be a priori given and made known to the transmitter, and the transmitter algorithms, again possibly under consideration of channel information, have to be a posteriori adapted correspondingly. Since the early times of radio communications, the rationale Tx orientation has been preferred because, seemingly, it has some kind of natural appeal to system designers. It was not before the 1990s that the first ideas of Rx orientation came up (cf. Table 1). It took another couple of years to clearly formulate this rationale in 2000 [1]. From then on, it attracted broader attention so that a systematical study could begin. This late perception of Rx ori- entation is astonishing because each of the two approaches, depending on the particular field of application, has its distinct pros. In the case of Tx orientation, the transmitter algorithms to be a priori determined can be chosen with a view to arrive at particularly simple transmitter implementations. On the other hand, in the case of Rx orientation, the receiver algorithms can be a priori determined in such a way that the receiver complexity is minimized. If we consider, as an important example of a radio transmission, mobile radio systems, the complexity of the mobile terminals (MT) should be as low as possible, whereas more complicated implementations can be tolerated at the base stations (BS). Having in mind the above-mentioned complexity features of the rationales Tx orientation and Rx orientation, this means that in the uplink (UL), the quasi natural choice would be Tx orientation, which leads to low-cost transmitters at the MTs, whereas in the downlink (DL), the rationale Rx orientation would be the favourite alternative because this results in simple receivers at the MTs. In $[1,2]$, the application of the rationale $\mathrm{Rx}$ orientation to mobile radio DLs is considered. 
TABLE 1: Selected early publications on Rx-oriented transmission in chronological order.

\begin{tabular}{|c|c|}
\hline References & $\begin{array}{l}\text { Type of system, proposed techniques, } \\
\text { and further remarks }\end{array}$ \\
\hline$[3,4]$ & $\begin{array}{l}\text { SISO, CDMA with spreading at Tx, design of FIR } \\
\text { prefilter (MF criterion) } \Rightarrow \text { Pre-Rake }\end{array}$ \\
\hline$[5]$ & $\begin{array}{l}\text { SISO, CDMA with spreading at Tx, pre-decorrelator } \\
\text { (ZF criterion) }\end{array}$ \\
\hline$[6]$ & $\begin{array}{l}\text { SISO, CDMA with spreading at Tx, pre-decorrelator } \\
\text { (ZF criterion) }\end{array}$ \\
\hline [7] & $\begin{array}{l}\text { SISO, CDMA with spreading at Tx, pre-decorrelator } \\
\text { (ZF criterion) and pre-MMSE (MMSE criterion) }\end{array}$ \\
\hline$[8]$ & $\begin{array}{l}\text { MISO, CDMA with spreading at Tx, design of FIR } \\
\text { prefilter (MF / ZF / MMSE criterion) } \Rightarrow \text { Pre-Rake }\end{array}$ \\
\hline [9] & $\begin{array}{l}\text { SISO, CDMA with spreading at Tx, design of FIR } \\
\text { prefilter (MF criterion) } \Rightarrow \text { Pre-Rake }\end{array}$ \\
\hline$[10]$ & MIMO, MMSE processing (MMSE criterion) \\
\hline$[11]$ & $\begin{array}{l}\text { MISO, CDMA, joint transmission (ZF criterion) } \\
\Rightarrow \text { TxZF }\end{array}$ \\
\hline$[12]$ & $\begin{array}{l}\text { MISO, CDMA, joint predistortion (ZF criterion) } \\
\Rightarrow \text { TxZF }\end{array}$ \\
\hline [13] & $\begin{array}{l}\text { SISO, CDMA with spreading at Tx, design of FIR } \\
\text { prefilter (ZF criterion) }\end{array}$ \\
\hline$[14]$ & $\begin{array}{l}\text { MISO, CDMA, joint transmission (ZF criterion) } \\
\Rightarrow \text { TxZF }\end{array}$ \\
\hline
\end{tabular}

As mentioned above, in the case of Tx orientation, channel knowledge would be desirable at the MTs, whereas in the case of Rx orientation, such knowledge should be available at the BSs. This means that, in the case of mobile radio systems, the above proposed combination of Tx orientation in the UL and Rx orientation in the DL is particularly easily feasible, if the utilized duplexing scheme is time division duplexing (TDD). In TDD, the UL and the DL use the same frequency in temporally separated periods so that, due to the reciprocity theorem, both links experience the same channel impulse responses as long as the time elapsing between UL and DL transmissions is not too large. Therefore, the channel knowledge needed by the BS receivers in the Tx-oriented UL and obtainable for instance based on the transmission of training signals by the MTs can be used also as the channel knowledge required for the Rx-oriented DL transmission. This approach to exploit channel knowledge available in the BS for DL transmission has the additional advantage that no resources have to be sacrificed for the transmission of training signals in the DL, which is, anyhow, capacity-wise the more critical one of the two links.

An important asset with respect to increasing the spectrum efficiency of radio transmission systems is the use of multiantennas instead of single antennas at both the transmitter and the receiver $[15,16]$. Such multi-antenna structures were given the designation multiple input multiple output (MIMO). A series of theoretical results concerning the capacity of MIMO systems $[17,18]$ and the implementation of such systems $[19,20]$ came up in recent years. The present paper has the goal to study and compare the rationales $\mathrm{Tx}$

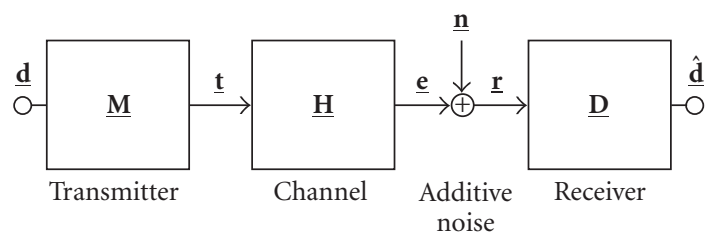

FIGURE 1: Generic model of a linear transmission system.

orientation and Rx orientation and to show some dualities and differences, if linear versions of these schemes are utilized in combination with MIMO antenna structures. Linear systems have, in contrast to nonlinear systems as for instance considered in [21], the advantage of lower complexity $[22,23]$. Nevertheless, also in linear systems, a beneficial nonlinear feature can be introduced by operating the linear inner MIMO system in combination with outer FEC coding at the transmitter and FEC decoding at the receiver.

In Section 2, a generic model of linear transmission systems is developed. The topic of Section 3 is the detailed description of the rationales $\mathrm{Tx}$ orientation and Rx orientation under inclusion of the linear algorithms to be applied at the transmitters and receivers. In this section, also the quantity signal-to-noise-plus-interference ratio (SNIR) suitable for performance of comparisons of the two rationales is introduced. The generic model developed in Section 2 and the findings of Section 3 are adapted to linear MIMO transmission systems in Section 4. Section 5 presents the results of system simulations; these results help to decide in which cases Tx orientation or Rx orientation should be chosen. Finally, Section 6 summarizes the paper.

The investigations are performed in the time-discrete equivalent low-pass domain under utilization of the vectormatrix representation of signals and system components [24]. Consequently, signals and channel impulse responses are represented by complex vectors or matrices which are printed in bold face. In the analysis, $[\cdot]_{n, n}$ designates the $n$th diagonal element of a square matrix in brackets, $[\cdot]_{n}$ stands for the $n$th row of a matrix in brackets or the $n$th element of a vector in brackets, and $\|\cdot\|_{2}$ denotes the Euclidean norm of the vector in brackets. Moreover, the operation $\overline{\operatorname{diag}}(\cdot)$ yields a copy of the matrix in brackets with the diagonal elements being set to zero.

\section{GENERIC MODEL OF LINEAR TRANSMISSION SYSTEMS}

Figure 1 shows the generic model of a linear transmission system. In this model, the transmitter, the channel, and the receiver are described by the matrices $\underline{\mathbf{M}}, \underline{\mathbf{H}}$, and $\underline{\mathbf{D}}$, respectively [1]. $\underline{\mathbf{M}}, \underline{\mathbf{H}}$, and $\underline{\mathbf{D}}$ are termed modulator matrix, channel matrix, and demodulator matrix, respectively. The signals occurring in the structure of Figure 1 are represented by the following column vectors:

(i) d: data signal to be transmitted,

(ii) t: transmit signal,

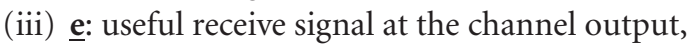


TABLE 2: Dimensions of the vectors and matrices used in the structure of Figure 1.

\begin{tabular}{cc}
\hline Vector or matrix, respectively & Dimensions \\
\hline$\underline{\mathbf{d}}=\left(\underline{d}_{1}, \ldots, \underline{d}_{N}\right)^{\mathrm{T}}$ & $\mathbb{C}^{N \times 1}$ \\
$\underline{\mathbf{M}}$ & $\mathbb{C}^{Q \times N}, Q \geq N$ \\
$\underline{\mathbf{t}}$ & $\mathbb{C}^{Q \times 1}$ \\
$\underline{\mathbf{H}}$ & $\mathbb{C}^{S \times Q}$ \\
$\underline{\mathbf{e}}$ & $\mathbb{C}^{S \times 1}$ \\
$\underline{\mathbf{n}}$ & $\mathbb{C}^{S \times 1}$ \\
$\underline{\mathbf{r}}$ & $\mathbb{C}^{S \times 1}$ \\
$\underline{\mathbf{D}}$ & $\mathbb{C}^{N \times S}$ \\
$\underline{\hat{\mathbf{d}}}$ & $\mathbb{C}^{N \times 1}$ \\
\hline
\end{tabular}

(iv) $\underline{\mathbf{n}}$ : Gaussian noise signal at the receiver input,

(v) r: disturbed signal at the receiver input,

(vi) $\underline{\hat{\mathbf{d}}}$ : linear estimate of $\underline{\mathbf{d}}$ at the receiver output.

The dimensions of the vectors and matrices used in the structure of Figure 1 are specified in Table 2.

The elements $\underline{d}_{n}, n=1, \ldots, N$, of $\underline{\mathbf{d}}$ are the data symbols to be transmitted and are taken from a finite symbol set

$$
\mathbb{V}=\left\{\underline{v}_{1} \cdots \underline{v}_{M}\right\}
$$

of cardinality $M . \underline{\mathbf{d}}$ and $\underline{\mathbf{n}}$ are assumed to be wide-sense stationary with zero mean and the covariance matrices

$$
\begin{gathered}
\underline{\mathbf{R}}_{\mathrm{d}}=2 \sigma_{\mathrm{d}}^{2} \mathbf{I}^{N \times N}, \\
\underline{\mathbf{R}}_{\mathrm{n}}=2 \sigma^{2} \mathbf{I}^{S \times S},
\end{gathered}
$$

respectively. In the system of Figure 1, the estimate $\underline{\hat{\mathbf{d}}}$ of $\underline{\mathbf{d}}$ obtained at the receiver output can be expressed as

$$
\begin{aligned}
& \underline{\hat{\mathbf{d}}}=\left(\underline{\hat{d}}_{1} \cdots \underline{\hat{d}}_{N}\right)^{\mathrm{T}}=\underline{\mathbf{D}} \underline{\mathbf{r}}=\underline{\mathbf{D}}(\underline{\mathbf{e}}+\underline{\mathbf{n}})=\underline{\mathbf{D}}(\underbrace{\underline{\mathbf{H}} \underline{\mathbf{t}}}_{\underline{\mathbf{e}}}+\underline{\mathbf{n}}) \\
& =\underline{\mathrm{D}}(\underline{\mathbf{H}} \underbrace{\mathbf{M}}_{\underline{\mathbf{M}}} \underline{\mathbf{d}}+\underline{\mathbf{n}})=\underline{\mathrm{D}} \underline{\mathbf{H}} \underline{\mathbf{M}} \underline{\mathbf{d}}+\underline{\mathrm{D}} \underline{\mathbf{n}} .
\end{aligned}
$$

$\underline{\mathrm{D}} \underline{\mathbf{H}} \underline{\mathbf{M}}$ is a square matrix of dimension $N \times N$. Generally, each data symbol $\underline{d}_{n}, n=1, \ldots, N$, has an influence on all $Q$ elements of $\underline{t}$. Therefore, $Q$ can be considered as a spreading factor, where, as we will see in Section 4, spreading can have a temporal and a spatial component.

According to (2) and (4), the mean radiated energy invested for the data symbol $\underline{d}_{n}$ becomes

$$
T_{n}=\frac{1}{2}\left\|\left[\underline{\mathbf{M}}^{\mathrm{T}}\right]_{n}\right\|_{2}^{2} 2 \sigma_{\mathrm{d}}^{2},
$$

where the factor " $1 / 2$ " results from the low-pass domain representation used within this contribution [25]. By averaging over all $N$ data symbols $\underline{d}_{n}, n=1, \ldots, N$, we obtain the mean radiated energy

$$
T=\frac{\sigma_{\mathrm{d}}^{2}}{N} \sum_{n=1}^{N}\left\|\left[\underline{\mathbf{M}}^{\mathrm{T}}\right]_{n}\right\|_{2}^{2}
$$

per data symbol.

The estimate $\underline{\hat{d}}_{n}$ of the transmitted data symbol $\underline{d}_{n}$ consists of the sum of a useful part

$$
\underline{d}_{\mathrm{useful}, n}=[\underline{\mathbf{D}} \underline{\mathbf{H}} \underline{\mathbf{M}}]_{n, n} \underline{d}_{n},
$$

of an interference part

$$
\underline{d}_{\text {int }, n}=[\overline{\operatorname{diag}}(\underline{\mathbf{D}} \underline{\mathbf{H}} \underline{\mathbf{M}}) \underline{\mathbf{d}}]_{n},
$$

and of a noise part

$$
\underline{d}_{\text {noise }, n}=[\underline{\mathbf{D}} \underline{\mathbf{n}}]_{n}
$$

see also [24]. In (8) and (9), the terms in brackets are column vectors. A concise and obvious quality measure for the estimates $\underline{\hat{d}}_{n}$ of (4) are the SNIRs $\gamma_{n}$ [24]. With (2), (3), (7), (8), and (9), we obtain

$$
\begin{aligned}
\gamma_{n} & =\frac{\mathrm{E}\left\{\left|\underline{d}_{\mathrm{useful}, n}\right|^{2}\right\}}{\mathrm{E}\left\{\left|\underline{d}_{\text {noise }, n}\right|^{2}\right\}+\mathrm{E}\left\{\left|\underline{d}_{\text {int }, n}\right|^{2}\right\}} \\
& =\frac{\left|[\underline{\mathbf{D}} \underline{\mathbf{H}} \underline{\mathbf{M}}]_{n, n}\right|^{2} \sigma_{\mathrm{d}}^{2}}{\left\|[\underline{\mathbf{D}}]_{n}\right\|_{2}^{2} \sigma^{2}+\left\|[\overline{\operatorname{diag}}(\underline{\mathbf{D}} \underline{\mathbf{H}} \underline{\mathbf{M}})]_{n}\right\|_{2}^{2} \sigma_{\mathrm{d}}^{2}} .
\end{aligned}
$$

Even though in this paper, $\gamma_{n}$ is adopted as the quality measure and quantitatively studied, ultimately the symbol error probabilities would be the proper measure. Fortunately, in many cases, noise plus interference can be modeled as white Gaussian noise with sufficient accuracy. Then, the error probabilities immediately follow from the values $\gamma_{n}$. Otherwise, also the probability density function of noise plus interference has to be taken into account.

\section{TRANSMITTER ORIENTATION AND RECEIVER ORIENTATION}

The a posteriori determination of $\underline{\mathrm{D}}$ in the case of linear Tx orientation or of $\underline{\mathbf{M}}$ in the case of linear Rx orientation have to be performed under the consideration of certain criteria. Depending on these criteria, different matrices $\underline{\mathbf{D}}$ or $\underline{\mathbf{M}}$, respectively, result. In what follows, first expressions for determining $\underline{\mathbf{D}}$ or $\underline{\mathbf{M}}$, respectively, are presented, and only then it will be explained which criteria stand behind these expressions. The authors believe that this procedure facilitates the understanding of the presentation, even though the said expressions are consequences of the related criteria.

In the case of Tx orientation, $\underline{\mathbf{M}}$ and $\underline{\mathbf{H}}$ are a priori given, whereas $\mathrm{D}$ is a posteriori determined at the Rx based on the knowledge of $\underline{\mathbf{M}}$ and $\underline{\mathbf{H}}$. Well-known approaches for determining $\underline{\mathrm{D}}$ are the receive matched filter $(\mathrm{RxMF})$, the receive 
zero forcer (RxZF), and the receive minimum mean square error estimator (RxMMSE) [24]. In these three cases, the demodulator matrix is a posteriori determined according to [24]

$$
\underline{\mathbf{D}}= \begin{cases}(\underline{\mathbf{H}} \underline{\mathbf{M}})^{\mathrm{H}} & (\mathrm{RxMF}), \\ {\left[(\underline{\mathbf{H}} \underline{\mathbf{M}})^{\mathrm{H}} \underline{\mathbf{H}} \underline{\mathbf{M}}\right]^{-1}(\underline{\mathbf{H}} \underline{\mathbf{M}})^{\mathrm{H}}} & (\mathrm{RxZF}), \\ {\left[(\underline{\mathbf{H}} \underline{\mathbf{M}})^{\mathrm{H}} \underline{\mathbf{H}} \underline{\mathbf{M}}+\sigma^{2} \mathbf{I}^{N \times N}\right]^{-1}(\underline{\mathbf{H}} \underline{\mathbf{M}})^{\mathrm{H}}} & (\mathrm{RxMMSE}) .\end{cases}
$$

In the case of Rx orientation, $\underline{\mathbf{H}}$ and $\underline{\mathrm{D}}$ are a priori given, and $\underline{M}$ is a posteriori determined at the Tx based on the knowledge of $\underline{\mathbf{H}}$ and $\underline{\mathbf{D}}$. Approaches meanwhile quite well known to determining $\underline{\mathbf{M}}$ are the transmit matched filter (TxMF) and the transmit zero forcer (TxZF) $[1,2]$. For these, the modulator matrix is a posteriori determined as follows:

$$
\underline{\mathbf{M}}= \begin{cases}(\underline{\mathbf{D}} \underline{\mathbf{H}})^{\mathrm{H}}, & (\mathrm{TxMF}) \\ (\underline{\mathbf{D}} \underline{\mathbf{H}})^{\mathrm{H}}\left[\underline{\mathbf{D}} \underline{\mathbf{H}}(\underline{\mathbf{D}} \underline{\mathbf{H}})^{\mathrm{H}}\right]^{-1} . & (\mathrm{TxZF})\end{cases}
$$

Other options for Rx orientation are various kinds of transmit minimum mean square error modulators (TxMMSE). In one version, which leads to a closed-form expression for $\underline{\mathbf{M}}$, we set out from a given average transmit energy $T$, see (6), and, under this condition, determine $\underline{\mathbf{M}}$ with a real scalar $k$ according to

$$
\begin{aligned}
& \underline{\mathbf{M}}=k(\underline{\mathbf{D}} \underline{\mathbf{H}})^{\mathrm{H}}\left[\underline{\mathbf{D}} \underline{\mathbf{H}}(\underline{\mathbf{D}} \underline{\mathbf{H}})^{\mathrm{H}}+\frac{\sigma^{2}}{N T} \operatorname{trace}\left(\underline{\mathbf{D}} \underline{\mathbf{D}}^{\mathrm{H}}\right) \mathbf{I}^{N \times N}\right]^{-1}, \\
& \text { s.t. } \quad \frac{\sigma_{\mathrm{d}}^{2}}{N} \sum_{n=1}^{N}\left\|\left[\underline{\mathbf{M}}^{\mathrm{T}}\right]_{n}\right\|_{2}^{2} \\
& \stackrel{!}{=} T \text { by proper choice of } k \quad \text { (TxMMSE). }
\end{aligned}
$$

Equation (13) was first published in [26] in a somewhat different form.

Now we come to the said criteria behind the expressions (11) to (13). The criterion being fulfilled by the Tx-oriented schemes of (11) and the Rx-oriented schemes of (12) is the maximization of $\gamma_{n}$ of (10) for a given mean transmit energy $T_{n}$ per data symbol $\underline{d}_{n}$, see (5), and under different side conditions, namely $[2,24]$, the following.

(1) RxMF, TxMF: the impact of the interference term $\left\|[\overline{\operatorname{diag}}(\underline{\mathbf{D}} \underline{\mathbf{H}} \underline{\mathbf{M}})]_{n}\right\|^{2} \sigma_{\mathrm{d}}^{2}$ in the denominator on the right-hand side of (10) is neglected.

(2) RxZF, TxZF: the impact of the interference term $\left\|[\overline{\operatorname{diag}}(\underline{\mathbf{D}} \underline{\mathbf{H}} \underline{\mathbf{M}})]_{n}\right\|^{2} \sigma_{\mathrm{d}}^{2}$ in the denominator on the right-hand side of (10) is eliminated by forcing this term to zero.

(3) RxMMSE: an optimum compromise between the impact of the noise term $\left\|[\underline{\mathbf{D}}]_{n}\right\|^{2} \sigma^{2}$ and the interference term $\left\|[\overline{\operatorname{diag}}(\underline{\mathbf{D}} \underline{\mathbf{H}} \underline{\mathbf{M}})]_{n}\right\|^{2} \sigma_{\mathrm{d}}^{2}$ is brought about.

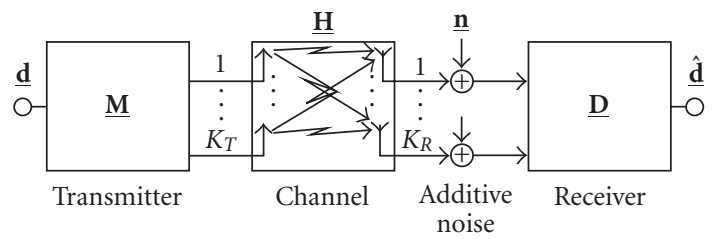

FIgURE 2: Linear MIMO transmission system.

In the case of the TxMMSE of (13), an average SNIR defined as

$$
\gamma_{\text {TxMMSE }}=\frac{\sigma_{\mathrm{d}}^{2} \sum_{n=1}^{N}\left|[\underline{\mathbf{D}} \underline{\mathbf{H}} \underline{\mathbf{M}}]_{n, n}\right|^{2}}{\sum_{n=1}^{N}\left\{\left\|[\underline{\mathbf{D}}]_{n}\right\|_{2}^{2} \sigma^{2}+\left\|[\overline{\operatorname{diag}}(\underline{\mathbf{D}} \underline{\mathbf{H}} \underline{\mathbf{M}})]_{n}\right\|_{2}^{2} \sigma_{\mathrm{d}}^{2}\right\}}
$$

is maximized for a given mean transmit energy $T$ of (6) [26].

An important issue when evaluating the transmission schemes of (11) to (13) is the determination of the SNIRs for given mean transmit energies $T_{n}$ of (5) or $T$ of (6). Therefore, the question arises how these energies can be predetermined. In the case of the Tx-oriented schemes of (11), the mean transmit energies $T_{n}$ per data symbol can be predetermined based on (5) when a priori establishing $\underline{\mathbf{M}}$ in a straightforward way. In the case of the TxMF and the TxZF, see (12), the predetermination of $T_{n}$ has to be accomplished as follows:

(i) determine $\underline{\mathbf{M}}$ by using (12),

(ii) column-wise scale this $\underline{\mathbf{M}}$ in such a way that (5) yields the desired mean energies $T_{n}$.

In the case of the TxMMSE, see (13), the mean radiated energy $T$ per data symbol can again be predetermined in a straightforward way.

The above theory is valid under the implicit understanding that the matrices to be inverted in (11) to (13) are nonsingular. This condition is usually fulfilled in reasonably designed systems. However, a closer look at this problem has yet to come.

\section{LINEAR MIMO TRANSMISSION SYSTEMS}

Figure 2 shows a linear MIMO transmission system with $K_{\mathrm{T}}$ antennas at the transmitter and $K_{\mathrm{R}}$ antennas at the receiver. The question is how in the case of such a MIMO system the vectors and matrices introduced in the generic transmission system of Section 2 have to be adjusted in order to make the equations derived in Sections 2 and 3 applicable.

We assume that each data symbol $\underline{d}_{n}$ is temporally spread over $Q_{t}$ chips [2]. Then, with the $K_{\mathrm{T}}$ matrices

$$
\underline{\mathbf{M}}^{\left(k_{\mathrm{T}}\right)}=\left(\begin{array}{cccc}
\underline{M}_{1,1}^{\left(k_{\mathrm{T}}\right)} & \underline{M}_{1,2}^{\left(k_{\mathrm{T}}\right)} & \cdots & \underline{M}_{1, N}^{\left(k_{\mathrm{T}}\right)} \\
\underline{M}_{2,1}^{\left(k_{\mathrm{T}}\right.} & \underline{M}_{2,2}^{\left(k_{\mathrm{T}}\right)} & \cdots & \underline{M}_{2, N}^{\left(k_{\mathrm{T}}\right)} \\
\vdots & \vdots & \ddots & \vdots \\
\underline{M}_{\mathrm{Q}_{\mathrm{t}}, 1}^{\left(k_{\mathrm{T}}\right.} & \underline{M}_{\mathrm{Q}_{\mathrm{t}}, 2}^{\left(k_{\mathrm{T}}\right)} & \cdots & \underline{M}_{\mathrm{Q}_{\mathrm{t}}, N}^{\left(k_{\mathrm{T}}\right)}
\end{array}\right) \in \mathbb{C}^{\mathrm{Q}_{\mathrm{t}} \times N}
$$


termed transmit antenna specific modulator matrices, the (total) modulator matrix takes the form [2]

$$
\begin{gathered}
\underline{\mathbf{M}}=\left(\underline{\mathbf{M}}^{(1)^{\mathrm{T}}} \underline{\mathbf{M}}^{(2)^{\mathrm{T}}} \cdots \underline{\mathbf{M}}^{\left(K_{\mathrm{T}}\right)^{\mathrm{T}}}\right)^{\mathrm{T}}, \\
\underline{\mathbf{M}} \in \mathbb{C}^{\left(Q_{\mathrm{t}} K_{\mathrm{T}}\right) \times N} .
\end{gathered}
$$

According to (16), the spreading factor $Q$ introduced in Table 2 now reads

$$
Q=Q_{\mathrm{t}} K_{\mathrm{T}}
$$

This shows that the total spreading quantified by $Q$ results from a temporal spreading and a spatial spreading represented by $Q_{\mathrm{t}}$ and $K_{\mathrm{T}}$, respectively.

The radio channel between transmit antenna $k_{\mathrm{T}}, k_{\mathrm{T}}=$ $1, \ldots, K_{\mathrm{T}}$, and receive antenna $k_{\mathrm{R}}, k_{\mathrm{R}}=1, \ldots, K_{\mathrm{R}}$, can be characterized by the transmit and receive antenna specific impulse response

$$
\underline{\mathbf{h}}^{\left(k_{\mathrm{R}}, k_{\mathrm{T}}\right)}=\frac{1}{W}\left(\underline{h}_{1}^{\left(k_{\mathrm{R}}, k_{\mathrm{T}}\right)} \underline{h}_{2}^{\left(k_{\mathrm{R}}, k_{\mathrm{T}}\right)} \cdots \underline{h}_{W}^{\left(k_{\mathrm{R}}, k_{\mathrm{T}}\right)}\right)^{\mathrm{T}}
$$

of dimension $W$ [2]. Taking into account that each of the $K_{\mathrm{T}}$ transmit antennas radiates a signal of dimension $Q_{\mathrm{t}} \times 1$, the signal transmission from the transmit antenna $k_{\mathrm{T}}, k_{\mathrm{T}}=$ $1, \ldots, K_{\mathrm{T}}$, to the receive antenna $k_{\mathrm{R}}, k_{\mathrm{R}}=1, \ldots, K_{\mathrm{R}}$, can be described by the transmit and receive antenna specific channel matrix

$$
\begin{aligned}
& \underline{\mathbf{H}}^{\left(k_{\mathrm{R}}, k_{\mathrm{T}}\right)}=\left(\begin{array}{cccc}
\underline{h}_{1}^{\left(k_{\mathrm{R}}, k_{\mathrm{T}}\right)} & 0 & \cdots & 0 \\
\underline{h}_{2}^{\left(k_{\mathrm{R}}, k_{\mathrm{T}}\right)} & \underline{h}_{1}^{\left(k_{\mathrm{R}}, k_{\mathrm{T}}\right)} & \ddots & \vdots \\
\vdots & \underline{h}_{2}^{\left(k_{\mathrm{R}}, k_{\mathrm{T}}\right)} & \ddots & 0 \\
\underline{h}_{W}^{\left(k_{\mathrm{R}}, k_{\mathrm{T}}\right)} & \vdots & \ddots & \underline{h}_{1}^{\left(k_{\mathrm{R}}, k_{\mathrm{T}}\right)} \\
0 & \underline{h}_{W}^{\left(k_{\mathrm{R}}, k_{\mathrm{T}}\right)} & \ddots & \underline{h}_{2}^{\left(k_{\mathrm{R}}, k_{\mathrm{T}}\right)} \\
\vdots & \ddots & \ddots & \vdots \\
0 & \cdots & 0 & \underline{h}_{W}^{\left(k_{\mathrm{R}}, k_{\mathrm{T}}\right)}
\end{array}\right), \\
& \underline{\mathbf{H}}^{\left(k_{\mathrm{R}}, k_{\mathrm{T}}\right)} \in \mathbb{C}^{\left(Q_{\mathrm{t}}+W-1\right) \times Q_{\mathrm{t}}}
\end{aligned}
$$

The $K_{\mathrm{R}} K_{\mathrm{T}}$ transmit and receive antenna specific channel matrices $\underline{\mathbf{H}}^{\left(k_{\mathrm{R}}, k_{\mathrm{T}}\right)}$ of (19) can be stacked to the (total) channel matrix

$$
\underline{\mathbf{H}}=\left(\begin{array}{cccc}
\underline{\mathbf{H}}^{(1,1)} & \underline{\mathbf{H}}^{(1,2)} & \cdots & \underline{\mathbf{H}}^{\left(1, K_{\mathrm{T}}\right)} \\
\underline{\mathbf{H}}^{(2,1)} & \underline{\mathbf{H}}^{(2,2)} & \cdots & \underline{\mathbf{H}}^{\left(2, K_{\mathrm{T}}\right)} \\
\vdots & \vdots & \ddots & \vdots \\
\underline{\mathbf{H}}^{\left(K_{\mathrm{R}}, 1\right)} & \underline{\mathbf{H}}^{\left(K_{\mathrm{R}}, 2\right)} & \cdots & \underline{\mathbf{H}}^{\left(K_{\mathrm{R}}, K_{\mathrm{T}}\right)}
\end{array}\right),
$$

According to (20), the quantity $S$ introduced in Table 2 can be expressed as

$$
S=\left(Q_{\mathrm{t}}+W-1\right) K_{\mathrm{R}}
$$

in the case of the considered MIMO system. Therefore, the signals $\underline{\mathbf{e}}, \underline{\mathbf{n}}$, and $\underline{\mathbf{r}}$, see Table 2 , have the dimension $\left[\left(Q_{t}+\right.\right.$ $\left.W-1) K_{\mathrm{R}}\right] \times 1$. Consequently,

$$
\underline{\mathbf{D}} \in \mathbb{C}^{N \times\left[\left(Q_{\mathrm{t}}+W-1\right) K_{\mathrm{R}}\right]}
$$

holds for the demodulator matrix.

With the matrices $\underline{\mathbf{M}}, \underline{\mathbf{H}}$, and $\underline{\mathbf{D}}$ defined by (16), (20), and (22), respectively, the different transmission schemes specified by (11), (12), and (13) can be immediately applied to linear MIMO transmission systems.

\section{SYSTEM EVALUATIONS BY SIMULATIONS}

Based on the performance measure SNIR of (10), different versions of linear MIMO transmission systems can be compared and assessed. Questions to be answered by such comparisons concern

(i) the performance difference of $\mathrm{Tx}$-oriented and $\mathrm{Rx}$ oriented systems,

(ii) the influence of the antenna numbers $K_{\mathrm{T}}$ and $K_{\mathrm{R}}$ on the system performance.

Because a closed-form analysis is not possible, these questions will be addressed by simulations in what follows. Concerning the design of linear MIMO transmission systems, besides the distinction between $\mathrm{Tx}$ orientation and $\mathrm{Rx}$ orientation, we can choose from a great variety of system parametrizations and channel realizations. In this paper, only a limited selection of such variants can be considered, which, nevertheless, will allow some generally valid statements. In all simulations, we set

$$
N=Q_{\mathrm{t}}=W=4 .
$$

Simulations are performed for different pairs $K_{\mathrm{T}}, K_{\mathrm{R}}$ of antenna numbers. For each such pair, many system realizations are investigated. In each realization, the elements of $\underline{\mathbf{h}}^{\left(k_{\mathrm{R}}, k_{\mathrm{T}}\right)}$ of (18) and-in the case of Tx orientation-the elements of $\underline{\mathbf{M}}$, or - in the case of Rx orientation - the elements of $\underline{\mathbf{D}}$ are chosen as independent realizations of a complex Gaussian random variable with variance 1 of its real and imaginary parts. For a given $T / \sigma^{2}$, by averaging over all $N$ values $\gamma_{n}$ of (10) and all realizations, the mean SNIR $\gamma$ can be obtained as a function of $T / \sigma^{2}$. Concerning the predetermination of $T$, see the last paragraph of Section 3. The determination of $\underline{\mathbf{h}}^{\left(k_{\mathrm{R}}, k_{\mathrm{T}}\right)}$ described above means that all $K_{\mathrm{T}} K_{\mathrm{R}}$ channel impulse responses are totally uncorrelated. The opposite to this extreme case would be totally correlated channel impulse responses, which, however, are not considered in this paper.

In Figures $3 \mathrm{a}, 3 \mathrm{~b}, 3 \mathrm{c}, 3 \mathrm{~d}, 3 \mathrm{e}$, and $3 \mathrm{f}$, the mean SNIR $\gamma$ is plotted versus $T / \sigma^{2}$ for different pairs $K_{\mathrm{T}}, K_{\mathrm{R}}$ and different transmission schemes. The curves in these figures allow the following conclusions.

(1) Both in the case of Tx orientation and Rx orientation, the MF outperforms the ZF for small values of $T / \sigma^{2}$, and the ZF outperforms the MF for large values of $T / \sigma^{2}$. See Figures $3 \mathrm{a}, 3 \mathrm{~b}, 3 \mathrm{c}$, and $3 \mathrm{~d}$. 


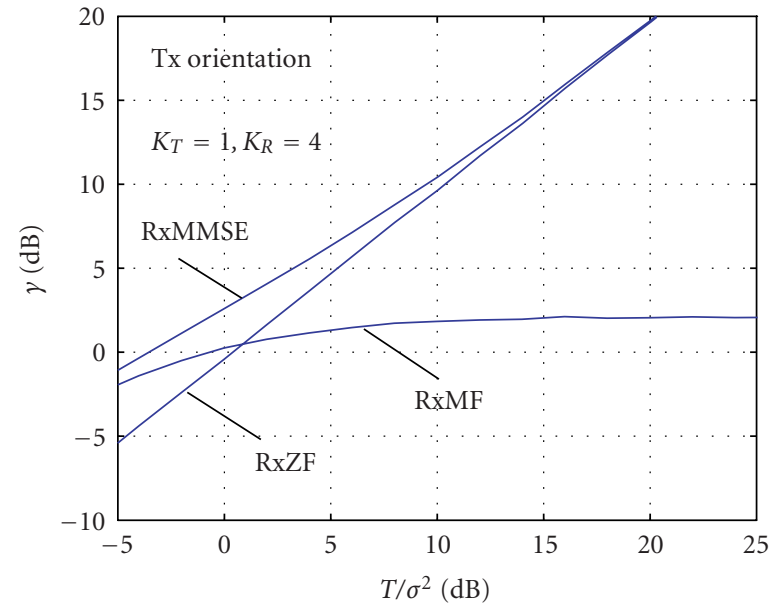

(a)

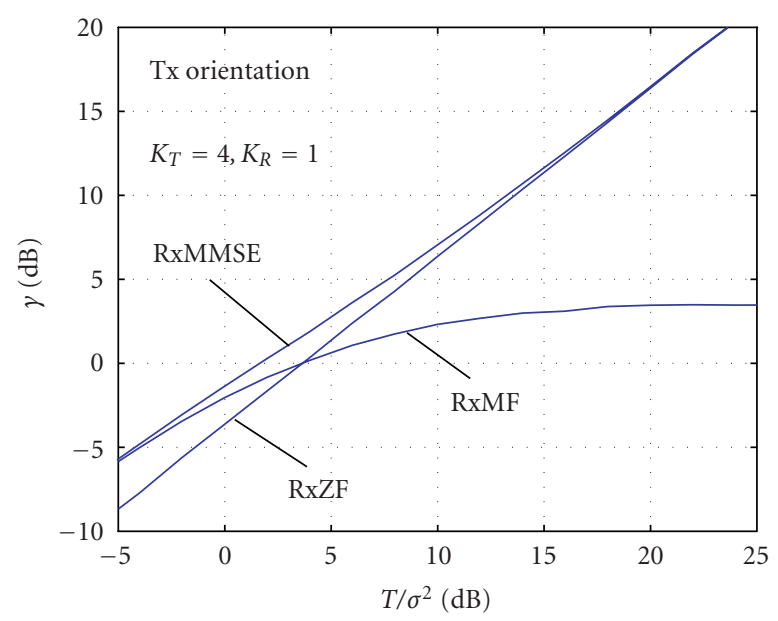

(c)

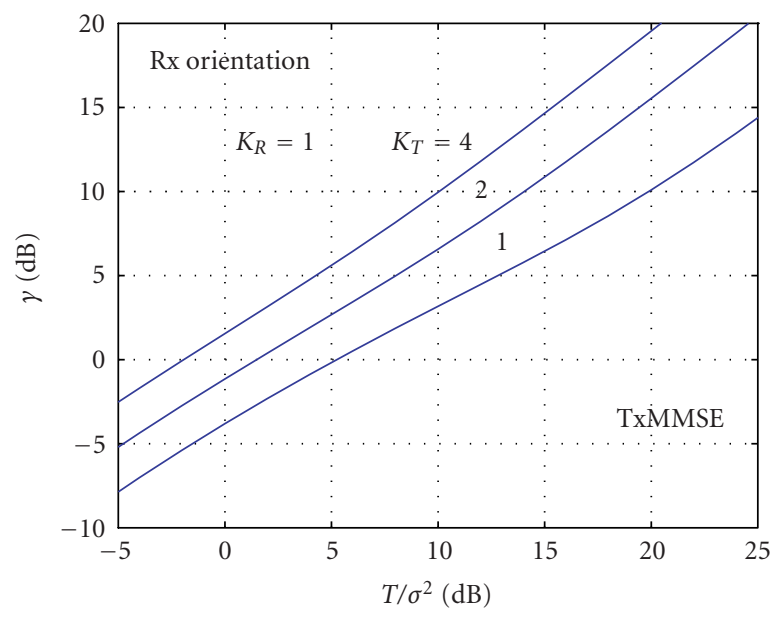

(e)

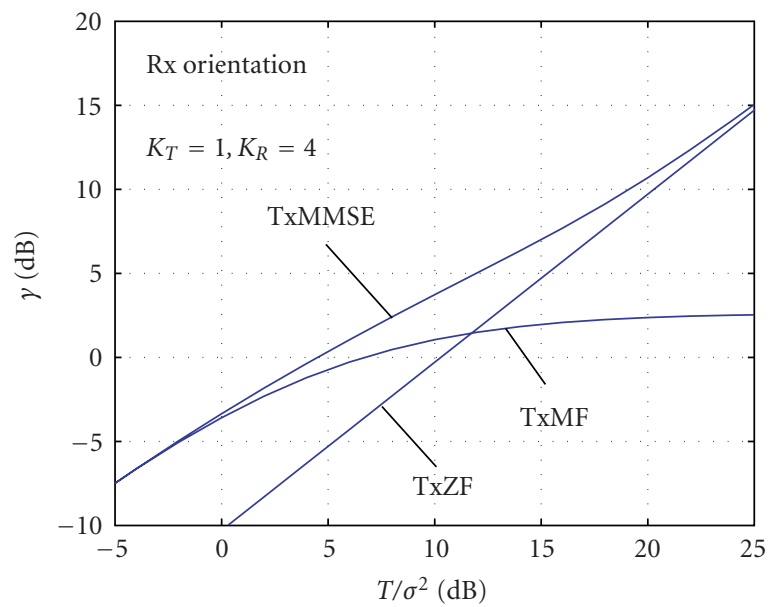

(b)

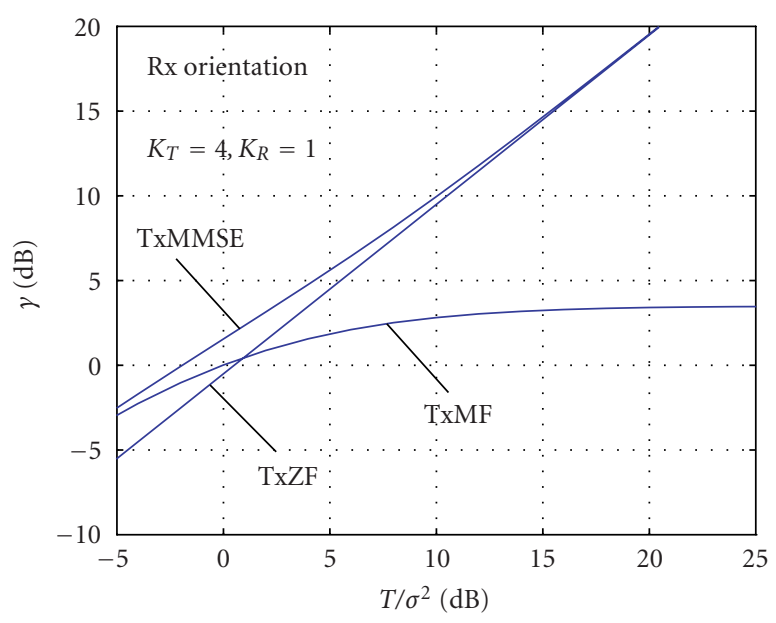

(d)

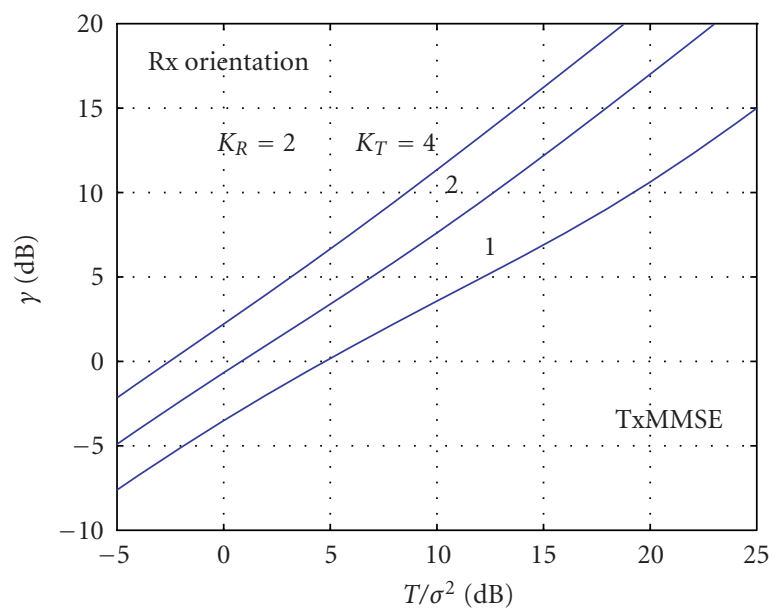

(f)

Figure 3: Mean SNIR $\gamma$ versus $T / \sigma^{2}$ for the rationales Tx orientation and Rx orientation and for different combinations $K_{\mathrm{T}}, K_{\mathrm{R}} ; N=Q_{\mathrm{t}}=$ $W=4$. 
(2) Both in the case of Tx orientation and Rx orientation, the MMSE outperforms the MF and the ZF. For small values of $T / \sigma^{2}$, the performance of the MMSE converges to the performance of the MF, and for large values of $T / \sigma^{2}$ to the performance of the ZF. See Figures $3 \mathrm{a}, 3 \mathrm{~b}, 3 \mathrm{c}$, and $3 \mathrm{~d}$.

(3) If the number $K_{\mathrm{R}}$ of receive antennas is larger than the number $K_{\mathrm{T}}$ of transmit antennas, Tx orientation should be chosen because it outperforms Rx orientation. If $K_{\mathrm{R}}$ is smaller than $K_{\mathrm{T}}$, the opposite is true. Compare Figures $3 \mathrm{a}$ and $3 \mathrm{~b}$, and Figures $3 \mathrm{c}$ and $3 \mathrm{~d}$.

(4) The performance is enhanced with growing $K_{\mathrm{T}}$ and $K_{\mathrm{R}}$. See Figures $3 \mathrm{e}$ and $3 \mathrm{f}$.

If we compare the Tx-oriented schemes for $K_{\mathrm{T}}=1$ and $K_{\mathrm{R}}=4$ (see Figure $3 \mathrm{a}$ ) with the $\mathrm{Rx}$-oriented schemes for $K_{\mathrm{T}}=4$ and $K_{\mathrm{R}}=1$ (see Figure $3 \mathrm{~d}$ ) or if we compare the Tx-oriented schemes for $K_{\mathrm{T}}=4, K_{\mathrm{R}}=1$ (see Figure 3c) with the Rx-oriented schemes for $K_{\mathrm{T}}=1, K_{\mathrm{R}}=4$ (see Figure $3 \mathrm{~b}$ ), we can find a very interesting result: if the number of antennas in the two considered schemes both at the a priori given sides and at the a posteriori adapted sides are equal, then the $\mathrm{Rx}$-oriented schemes perform worse than the $\mathrm{Tx}$ oriented schemes. This effect results from the assumption of totally uncorrelated channel impulse responses of dimension $W$, which is larger than one.

\section{SUMMARY}

A system model for linear MIMO transmission systems is developed, and this model is worked out for the cases of Tx-oriented and Rx-oriented systems. Based on the system model, performance comparisons and evaluations are made in which the performance measure is the mean SNIR, and the recommendations concerning the system design are given.

\section{ACKNOWLEDGMENTS}

The authors gratefully appreciate the fruitful exchange of ideas with C. A. Jötten, H. Tröger, and T. Weber from the Research Group for RF Communications, University of Kaiserslautern (UKL). The support of individual parts of this work in the framework of the EU-IST-Project FLOWS (Flexible Convergence of Wireless Standards and Services), by DFG, by Siemens AG, and by the supercomputer staff of the central computer facility (RHRK) of the TUKL is highly acknowledged. Thanks are also extended to the anonymous reviewers for their valuable comments and to A. Bruhn and M. Cuntz for, despite all time pressure, carefully typesetting the manuscript in $\mathrm{ET}_{\mathrm{E}} \mathrm{X}$.

\section{REFERENCES}

[1] P. W. Baier, M. Meurer, T. Weber, and H. Tröger, "Joint transmission (JT), an alternative rationale for the downlink of time division CDMA using multi-element transmit antennas," in Proc. IEEE 6th International Symposium on Spread Spectrum Techniques and Applications (ISSSTA '00), vol. 1, pp. 1-5, Parsippany, NJ, USA, September 2000.
[2] P. W. Baier, W. Qiu, H. Tröger, C. A. Jötten, and M. Meurer, "Modelling and optimization of receiver oriented multi-user MIMO downlinks for frequency selective channels," in Proc. 10th International Conference on Telecommunications (ICT '03), vol. 2, pp. 1547-1554, Papeete, French Polynesia, February 2003.

[3] R. Esmailzadeh and M. Nakagawa, "Pre-RAKE diversity combination for direct sequence spread spectrum mobile communications systems," IEICE Transactions on Communications, vol. 76, no. 8, pp. 1008-1015, 1993.

[4] R. Esmailzadeh, E. Sourour, and M. Nakagawa, "Pre-RAKE diversity combining in time division duplex CDMA mobile communications," in Proc. IEEE 6th International Symposium on Personal, Indoor and Mobile Radio Communications (PIMRC '95), vol. 2, pp. 431-435, Toronto, Ontario, Canada, September 1995.

[5] Z. Tang and S. Cheng, "Interference cancellation for DS-CDMA systems over flat fading channels through predecorrelating," in Proc. IEEE 5th International Symposium on Personal, Indoor and Mobile Radio Communications (PIMRC '94), vol. 2, pp. 435-438, The Hague, The Netherlands, September 1994.

[6] H. Matsutani, Y. Sanada, and M. Nakagawa, "A forward link intracell orthogonalization technique using multicarrier predecorrelation for CDMA wireless local communication system," in Proc. IEEE 8th International Symposium on Personal, Indoor and Mobile Radio Communications (PIMRC'97), vol. 1, pp. 125-129, Helsinki, Finland, September 1997.

[7] B. Vojcic and W. M. Jang, "Transmitter precoding in synchronous multiuser communications," IEEE Transactions on Communications, vol. 46, no. 10, pp. 1346-1355, 1998.

[8] G. Montalbano, I. Ghauri, and D. T. M. Slock, "Spatiotemporal array processing for CDMA/SDMA downlink transmission," in Proc. 32nd Asilomar Conference on Signals, Systems and Computers, vol. 2, pp. 1337-1341, Pacific Grove, Calif, USA, November 1998.

[9] A. N. Barreto and G. Fettweis, "On the downlink capacity of TDD CDMA systems using a Pre-RAKE," in IEEE Global Telecommunications Conference (GLOBECOM '99), vol. 1A, pp. 117-121, Rio de Janeiro, Brazil, December 1999.

[10] H. R. Karimi, M. Sandell, and J. Salz, "Comparison between transmitter and receiver array processing to achieve interference nulling and diversity," in Proc. IEEE 10th International Symposium on Personal, Indoor and Mobile Radio Communications (PIMRC'99), vol. 3, pp. 997-1001, Osaka, Japan, September 1999.

[11] M. Meurer, P. W. Baier, T. Weber, Y. Lu, and A. Papathanassiou, "Joint transmission: advantageous downlink concept for CDMA mobile radio systems using time division duplexing," IEE Electronics Letters, vol. 11, no. 10, pp. 900-901, 2000.

[12] F. Kowalewski and P. Mangold, "Joint predistortion and transmit diversity," in Proc. IEEE Global Telecommunications Conference (GLOBECOM '00), vol. 1, pp. 245-249, San Francisco, Calif, USA, 2000.

[13] M. Brandt-Pearce and A. Dharap, "Transmitter-based multiuser interference rejection for the down-link of a wireless CDMA system in a multipath environment," IEEE Journal on Selected Areas in Communications, vol. 18, no. 3, pp. 407-417, 2000.

[14] M. Joham and W. Utschick, "Downlink processing for mitigation of intracell interference in DS-CDMA systems," in Proc. IEEE 6th International Symposium on Spread Spectrum Techniques and Applications (ISSSTA '00), vol. 1, pp. 15-19, Parsippany, NJ, USA, September 2000. 
[15] G. G. Raleigh and J. M. Cioffi, "Spatio-temporal coding for wireless communication," IEEE Transactions on Communications, vol. 46, no. 3, pp. 357-366, 1998.

[16] D. Gesbert, M. Shafi, D.-S. Shiu, P. J. Smith, and A. Naguib, "From theory to practice: an overview of MIMO space-time coded wireless systems," IEEE Journal on Selected Areas in Communications, vol. 21, no. 3, pp. 281-302, 2003.

[17] G. J. Foschini and M. Gans, "On limits of wireless communications in a fading environment when using multiple antennas," Wireless Personal Communications, vol. 6, no. 3, pp. 311-335, 1998.

[18] I. E. Telatar, "Capacity of multi-antenna Gaussian channels," European Transactions on Telecommunications, vol. 10, no. 6, pp. 585-595, 1999.

[19] G. J. Foschini, "Layered space-time architecture for wireless communication in a fading environment when using multielement antennas," Bell Labs Technical Journal, vol. 1, no. 2, pp. 41-59, 1996.

[20] P. Wolniansky, G. J. Foschini, G. D. Golden, and R. A. Valenzuela, "V-BLAST: An architecture for realizing very high data rates over the rich-scattering wireless channel," in Proc. URSI International Symposium on Signals, Systems, and Electronics (ISSSE '98), pp. 295-300, Pisa, Italy, 1998.

[21] E. Biglieri, G. Taricco, and A. Tulino, "Decoding space-time codes with BLAST architectures," IEEE Transactions on Signal Processing, vol. 50, no. 10, pp. 2547-2552, 2002.

[22] T. Weber and M. Meurer, "Optimum joint transmission: Potentials and dualities," in Proc. 6th International Symposium on Wireless Personal Multimedia Communications (WPMC '03), vol. 1, pp. 79-83, Yokosuka, Japan, October 2003.

[23] R. Irmer, W. Rave, and G. Fettweis, "Minimum BER transmission for TDD-CDMA in frequency-selective channels," in Proc. IEEE 14th International Symposium on Personal, Indoor and Mobile Radio Communications (PIMRC '03), vol. 2, pp. 1260-1264, Beijing, China, September 2003.

[24] A. Klein, Multi-User Detection of CDMA Signals - Algorithms and Their Application to Cellular Mobile Radio, Number 423 in Fortschrittberichte VDI, Reihe 10. VDI-Verlag, Düsseldorf, Germany, 1996.

[25] S. Stein and J. J. Jones, Modern Communication Principles, McGraw-Hill, New York, NY, USA, 1967.

[26] M. Joham, K. Kusume, M. H. Gzara, W. Utschick, and J. A. Nossek, "Transmit Wiener filter for the downlink of TDD DSCDMA systems," in Proc. IEEE 7th International Symposium on Spread Spectrum Techniques and Applications (ISSSTA '02), vol. 1, pp. 9-13, Prague, Czech Republic, September 2002.

Michael Meurer was born in Dernbach (Westerwald), Germany, in 1974 and received the diploma in electrical engineering in 1998 and the doctoral degree in 2003, both from the University of Kaiserslautern, Germany. After graduation in October 1998, he joined the Research Group for RF Communications at the University of Kaiserslautern, Germany, as a Research Engineer, where he is presently active as a

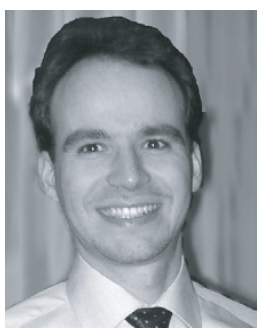
Senior Research Engineer and Senior Lecturer. His research interests are MIMO systems, receiver-oriented (joint transmission) and channel-oriented (joint transmitter and receiver optimization) transmission concepts, multiuser detection, and statistical signal processing. He is a Member of VDE/ITG and of the IEEE.
Paul Walter Baier was born in Backnang, Germany, in 1938, and graduated from the Technical University Munich, Germany. In 1970, he joined Siemens AG, Munich, where he was engaged in various topics of communications engineering. Since 1973, he has been a Professor for electrical communications and Director of the Institute for RF Communications and Fundamentals of Electronic Engineering at the University of

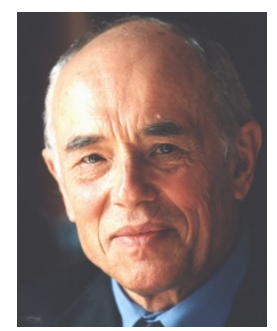
Kaiserslautern, Germany. His main research interests are spread spectrum techniques, impulse compression and synthetic aperture radars, mobile radio systems, and adaptive antennas. The basics of the TD-CDMA component of the UMTS Terrestrial Radio Access System (UTRA) agreed upon by 3GPP were developed by him and his coworkers in cooperation with Siemens and in the framework of EU projects. He is a member of VDE/ITG, of the URSI Member Committee Germany, and a Fellow of the IEEE. He was a Scholar of the Japanese Society for the Promotion of Science in 1997 and was awarded the Innovation Prize of the Mannesmann Mobile Radio Foundation in 1999 and the Ring of Honor of VDE Association for Electrical, Electronic \& Information Technologies in 2000. Since July 2002, he holds an honorary doctorate of the Technical University Munich.

Wei Qiu was born in Jiangsu, China, in 1975. He received his B.E. degree from Tsinghua University, Beijing, China, in 1999, and his M.S. degree from University of Kaiserslautern, Kaiserslautern, Germany, in 2001, both in electrical engineering. Since 2001, he has been a Research Engineer with the Research Group for RF Communications, the University of Kaiserslautern. His research interests are mainly concentrated

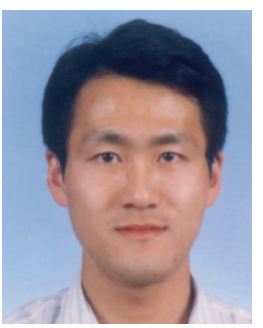
on mobile radio communications and on MIMO systems. He is a Student Member of IEEE. 